Article

\title{
Explicit Formulas for All Scator Holomorphic Functions in the (1+2)-Dimensional Case
}

\author{
Jan L. Cieśliński *(D) and Dzianis Zhalukevich (D) \\ Wydział Fizyki, Uniwersytet w Białymstoku, ul. Ciołkowskiego 1L, 15-245 Białystok, Poland; \\ den.zhal@yandex.by \\ * Correspondence: j.cieslinski@uwb.edu.pl
}

Received: 10 August 2020; Accepted: 16 September 2020; Published: 20 September 2020

\begin{abstract}
Scators form a vector space endowed with a non-distributive product, in the hyperbolic case, have physical applications related to some deformations of special relativity (breaking the Lorentz symmetry) while the elliptic case leads to new examples of hypercomplex numbers and related notions of holomorphicity. Until now, only a few particular cases of scator holomorphic functions have been found. In this paper we obtain all solutions of the generalized Cauchy-Riemann system which describes analogues of holomorphic functions in the $(1+2)$-dimensional scator space.
\end{abstract}

Keywords: scators; holomorphic functions; generalized Cauchy-Riemann equations

\section{Introduction}

Scators, as defined by Manuel Fernández-Guasti and Felipe Zaldívar [1], form a linear space with a specific multiplicative structure. In fact, we have two different structures: elliptic and hyperbolic. Namely, in the elliptic case, the scator product of scators $\stackrel{o}{a}:=\left(a_{0} ; a_{1}, \ldots, a_{n}\right)$ and $\stackrel{o}{b}:=\left(b_{0} ; b_{1}, \ldots, b_{n}\right)$ is given by $\stackrel{o}{u}:=\left(u_{0} ; u_{1}, \ldots, u_{n}\right)$, where

$$
\begin{aligned}
& u_{0}=a_{0} b_{0} \prod_{k=1}^{n}\left(1-\frac{a_{k} b_{k}}{a_{0} b_{0}}\right), \\
& u_{k}=\frac{a_{k} b_{0}+b_{k} a_{0}}{a_{0} b_{0}-a_{k} b_{k}} u_{0} \quad(k=1, \ldots, n),
\end{aligned}
$$

provided that $a_{0} \neq 0$ and $b_{0} \neq 0$ (more general case is presented and discussed in [1]). In the hyperbolic case, the formula is very similar (all minuses are replaced by pluses). In principle, one can consider mixed cases as well. The scator product is non-distributive, although a distributive approach has been proposed recently [2,3]. The so-called restricted space (defined by $a_{0}^{2}>a_{k}^{2}$ for $k=1, \ldots, n$ ) is an abelian group with respect to the scator product.

In the hyperbolic case, scators have potential physical applications related to generalizations of the special theory of relativity (breaking the Lorentz symmetry) [4,5]. The elliptic case is an interesting new example of (non-distributive) hypercomplex numbers [6].

Any hypercomplex numbers, like quaternions or Clifford numbers, lead to a natural question of defining and finding anlogues of holomorphic functions. In this paper, following [7], we focus on the most straightforward definition of holomorphicity, i.e., existence, at any point, of a direction-independent derivative. Fernández-Guasti derived a system of partial differential equations 
which assures scator differentiabiliy of this kind [7]. They can be considered as a generalization of Cauchy-Riemann equations of standard complex analysis:

$$
\begin{aligned}
& \frac{\partial u_{0}}{\partial x_{0}}=\frac{\partial u_{j}}{\partial x_{j}}, \quad \frac{\partial u_{j}}{\partial x_{0}}=-\frac{\partial u_{0}}{\partial x_{j}}, \\
& \frac{\partial u_{0}}{\partial x_{j}} \frac{\partial u_{0}}{\partial x_{m}}=-\frac{\partial u_{j}}{\partial x_{j}} \frac{\partial u_{j}}{\partial x_{m}}
\end{aligned}
$$

for all $m \neq j$, where $m$ and $j$ take values from 1 to $n$. Note that the last (nonlinear) equations appear only for $n>1$. The generalized Cauchy-Riemann Equation (2) consists of a set of linear equations ( $n$ copies of the Cauchy-Riemann equations, in fact) and a set of nonlinear equations (for $n>1$ ). The latter is the main difference with the standard case of complex holomorphic functions (i.e., the case $n=1$ ).

In this paper, we are going to solve the open problem of finding all solutions of the system (4) in the case $n=2$. Until now only two particular solutions were reported: four-parameter family of linear affine functions [7] and components exponential function [8].

\section{Generalized Cauchy-Riemann System in the Case $n=2$}

In this paper, we confine ourselves to the elliptic scator space of dimension $1+2$. We introduce the notation:

$$
x_{0}=x, \quad x_{1}=y, \quad x_{2}=z, \quad u_{0}=u, \quad u_{1}=v, \quad u_{2}=w .
$$

In the new notation, the generalized Cauchy-Riemann system (2) takes the following form

$$
\begin{aligned}
& u_{x}=v_{y}, \quad u_{y}=-v_{x}, \\
& u_{x}=w_{z}, \quad u_{z}=-w_{x}, \\
& u_{y} u_{z}=-v_{y} v_{z}=-w_{y} w_{z}
\end{aligned}
$$

where here and throughout the rest of this paper the subscripts $x, y, z$ mean partial derivative with respect to the corresponding variable.

Theorem 1. The full set of solutions to the generalized Cauchy-Riemann Equation (4) consists of three families.

- Components exponential functions

$$
\begin{aligned}
& u=q_{0}+p_{0} e^{k_{0} x} \cos \left(k_{0} y+k_{1}\right) \cos \left(k_{0} z+k_{2}\right), \\
& v=q_{1}+p_{0} e^{k_{0} x} \sin \left(k_{0} y+k_{1}\right) \cos \left(k_{0} z+k_{2}\right), \\
& w=q_{2}+p_{0} e^{k_{0} x} \cos \left(k_{0} y+k_{1}\right) \sin \left(k_{0} z+k_{2}\right) .
\end{aligned}
$$

- Linear functions

$$
\begin{aligned}
& u=b_{0}+a_{0}\left(x+f_{0} y+g_{0} z\right), \\
& v=b_{1}+a_{0}\left(y-f_{0} x-f_{0} g_{0} z\right), \\
& w=b_{2}+a_{0}\left(z-g_{0} x-f_{0} g_{0} y\right) .
\end{aligned}
$$

- Exceptional solutions

$$
\begin{aligned}
& u=c_{0} y+c_{1}, \quad v=-c_{0} x+V_{1}(z), \quad w=W_{1}(z), \\
& u=d_{0} z+d_{1}, \quad v=V_{2}(y), \quad w=-d_{0} x+W_{2}(z),
\end{aligned}
$$


where $q_{0}, q_{1}, q_{2}, p_{0}, k_{0}, k_{1}, k_{2}, b_{0}, b_{1}, b_{2}, a_{0}, f_{0}, g_{0}, c_{0}, c_{1}, d_{0}$ and $d_{1}$ are real constants $\left(p_{0} \neq 0, k_{0} \neq 0\right)$, and $V_{1}, V_{2}, W_{1}$ and $W_{2}$ are arbitrary functions of one variable.

The above set of solutions is not very rich, but one has to remember that in the case of quaternionic analysis the analogous set is much narrower and consists only of linear affine functions $[9,10]$. Therefore the quaternionic analysis, like the Clifford analysis, has to use other definitions of holomorphicity, see, e.g., [11].

In next sections, we present the proof of Theorem 1, by straightforward derivation of all solutions. It is convenient to divide the computation into three cases related to vanishing of the first and second $x$-derivatives of $u$.

\section{Components Exponential Functions}

This is the case characterized by

$$
u_{x} \not \equiv 0 \quad \text { and } \quad u_{x x} \not \equiv 0 .
$$

We are going to express the variables $v$ and $w$ in terms of $u$ and its derivatives:

$$
\begin{aligned}
& v_{x}=-u_{y}, \quad v_{y}=u_{x}, \quad v_{z}=u_{y} u_{z} / u_{x}, \\
& w_{x}=-u_{z}, \quad w_{z}=u_{x}, \quad w_{y}=-u_{y} u_{z} / u_{x} .
\end{aligned}
$$

Necessary conditions for the existence of $v$ and $w$ (provided that $u$ is known) are given by:

$$
\begin{aligned}
& v_{x y}=v_{y x}, \quad v_{x z}=v_{z x}, \quad v_{y z}=v_{z y}, \\
& w_{x y}=w_{y x}, \quad w_{x z}=w_{z x}, \quad w_{y z}=w_{z y} .
\end{aligned}
$$

Rewriting compatibility conditions (10) in terms of $u$ (and its derivatives), we obtain:

$$
\begin{aligned}
& u_{x x}+u_{y y}=0, \\
& u_{y z}-\left(u_{y} u_{z} / u_{x}\right)_{x}=0, \\
& u_{x z}+\left(u_{y} u_{z} / u_{x}\right)_{y}=0, \\
& u_{z y}-\left(u_{y} u_{z} / u_{x}\right)_{x}=0, \\
& u_{z z}+u_{x x}=0, \\
& u_{x y}+\left(u_{y} u_{z} / u_{x}\right)_{z}=0 .
\end{aligned}
$$

In other words, removing a redundant equation, we have:

$$
\begin{aligned}
& u_{y y}=u_{z z}=-u_{x x}, \\
& u_{y z} u_{x}^{2}=u_{x y} u_{x} u_{z}+u_{x z} u_{x} u_{y}-u_{y} u_{z} u_{x x}, \\
& u_{x z} u_{x}^{2}=-u_{y y} u_{x} u_{z}-u_{y z} u_{x} u_{y}+u_{y} u_{z} u_{x y}, \\
& u_{x y} u_{x}^{2}=-u_{y z} u_{x} u_{z}-u_{z z} u_{x} u_{y}+u_{y} u_{z} u_{x z},
\end{aligned}
$$

or, in the matrix form:

$$
\left(\begin{array}{ccc}
u_{x} u_{z} & u_{x} u_{y} & -u_{x}^{2} \\
-u_{y} u_{z} & u_{x}^{2} & u_{x} u_{y} \\
u_{x}^{2} & -u_{y} u_{z} & u_{x} u_{z}
\end{array}\right)\left(\begin{array}{l}
u_{x y} \\
u_{x z} \\
u_{y z}
\end{array}\right)=u_{x x}\left(\begin{array}{l}
u_{y} u_{z} \\
u_{x} u_{z} \\
u_{x} u_{y}
\end{array}\right)
$$


where $u_{y y}$ and $u_{z z}$ were replaced by $-u_{x x}$. This equation can be solved by inverting the matrix on the left-hand side:

$$
\left(\begin{array}{c}
u_{x y} \\
u_{x z} \\
u_{y z}
\end{array}\right)=\frac{u_{x x}}{u_{x}^{2}\left(u_{x}^{2}+u_{y}^{2}\right)\left(u_{x}^{2}+u_{z}^{2}\right)}\left(\begin{array}{ccc}
u_{x} u_{z}\left(u_{x}^{2}+u_{y}^{2}\right) & 0 & u_{x}^{2}\left(u_{x}^{2}+u_{y}^{2}\right) \\
u_{x} u_{y}\left(u_{x}^{2}+u_{z}^{2}\right) & u_{x}^{2}\left(u_{x}^{2}+u_{z}^{2}\right) & 0 \\
u_{y}^{2} u_{z}^{2}-u_{x}^{4} & u_{x} u_{y}\left(u_{x}^{2}+u_{z}^{2}\right) & u_{x} u_{z}\left(u_{x}^{2}+u_{y}^{2}\right)
\end{array}\right)\left(\begin{array}{l}
u_{y} u_{z} \\
u_{x} u_{z} \\
u_{x} u_{y}
\end{array}\right) .
$$

Now, the right-hand side turns out to be surprisingly simple and Equation (14) is equivalent to the following system of three equations:

$$
u_{x y}=\frac{u_{y}}{u_{x}} u_{x x}, \quad u_{x z}=\frac{u_{z}}{u_{x}} u_{x x}, \quad u_{y z}=\frac{u_{y} u_{z}}{u_{x}^{2}} u_{x x} .
$$

The first two equations can be expressed as conservation laws and, then, easily solved:

$$
\begin{aligned}
& \frac{d}{d x}\left(\frac{u_{y}}{u_{x}}\right)=0 \quad \Longrightarrow \quad u_{y}=f(y, z) u_{x}, \\
& \frac{d}{d x}\left(\frac{u_{z}}{u_{x}}\right)=0 \quad \Longrightarrow \quad u_{z}=g(y, z) u_{x},
\end{aligned}
$$

where $f$ and $g$ are some functions of two variables. Substituting (16) into Equation (15) we obtain

$$
u_{x y}=f u_{x x}, \quad u_{x z}=g u_{x x}, \quad u_{y z}=f g u_{x x} .
$$

Differentiating (16) with respect to $z$ and $y$, respectively, we get

$$
u_{y z}=f_{z} u_{x}+f u_{x z}, \quad u_{z y}=g_{y} u_{x}+g u_{x y} .
$$

Then, using (17), we obtain $f_{z}=0$ and $g_{y}=0$, i.e.,

$$
f=f(y), \quad g=g(z) .
$$

Now, differentiating (16) with respect to $y$ and $z$, respectively, we get

$$
\begin{aligned}
& f_{y} u_{x}+f^{2} u_{x x}=-u_{x x} \\
& g_{z} u_{x}+g^{2} u_{x x}=-u_{x x},
\end{aligned}
$$

where we took into account $u_{y y}=-u_{x x}$ and $u_{z z}=-u_{x x}$. Therefore:

$$
\frac{f_{y}}{1+f^{2}}=\frac{g_{z}}{1+g^{2}}=-\frac{u_{x x}}{u_{x}} .
$$

Thus, by virtue of (19), we have

$$
\frac{f_{y}}{1+f^{2}}=-k_{0}, \quad \frac{g_{z}}{1+g^{2}}=-k_{0}, \quad u_{x x}=k_{0} u_{x},
$$

where $k_{0}=$ const. In this section, due to the condition (8), we confine ourselves to $k_{0} \neq 0$. Then

$$
\begin{aligned}
& k_{0} \neq 0 \Longrightarrow u=p(y, z) e^{k_{0} x}+q(y, z), \\
& f=-\tan \left(k_{0} y+k_{1}\right), \quad g=-\tan \left(k_{0} z+k_{2}\right),
\end{aligned}
$$


where $p$ and $q$ are functions of two variables and $k_{1}$ and $k_{2}$ are constants. Then, Equation (16) imply

$$
\begin{aligned}
& q_{y}=q_{z}=0 \quad \Longrightarrow \quad q=q_{0}=\text { const }, \\
& p_{y}=k_{0} p f, \quad p_{z}=k_{0} p g .
\end{aligned}
$$

The last two equations can be solved, yielding

$$
p=p_{0} \cos \left(k_{0} y+k_{1}\right) \cos \left(k_{0} z+k_{2}\right),
$$

where $p_{0}=$ const. Hence, $u$ is proved to be of the form (5). Note that now the equations from the first line of (12) are identically satisfied. Finally, the system (9) takes the form

$$
\begin{array}{ll}
v_{x}=k_{0} e^{k_{0} x} \sin \left(k_{0} y+k_{1}\right) \cos \left(k_{0} z+k_{2}\right), & v_{y}=w_{z}=k_{0} e^{k_{0} x} \cos \left(k_{0} y+k_{1}\right) \cos \left(k_{0} z+k_{2}\right), \\
w_{x}=k_{0} e^{k_{0} x} \cos \left(k_{0} y+k_{1}\right) \sin \left(k_{0} z+k_{2}\right), & v_{z}=w_{y}=k_{0} e^{k_{0} x} \sin \left(k_{0} y+k_{1}\right) \sin \left(k_{0} z+k_{2}\right),
\end{array}
$$

and its only solution is given by the last two equations of (5). Special case $k_{1}=k_{2}=q_{0}=q_{1}=q_{2}=0$ and $k_{0}=p_{0}=1$, known as components exponential function, was shown to be differentiable (i.e., scator holomorphic) earlier, see [8], Lemma 2.

\section{Linear Functions}

Linear functions satisfying (4) can be obtained directly, by substituting a linear ansatz and computing its coefficients. However, in order to obtain all solutions to (4), we follow the pattern of the previous section, now assuming:

$$
u_{x} \not \equiv 0, \quad \text { and } \quad u_{x x} \not \equiv 0 .
$$

Then, the third equation of (22) implies $k_{0}=0$.

$$
k_{0}=0 \quad \Longrightarrow \quad u_{x x}=0, u_{y y}=0, u_{z z}=0 \quad \Longrightarrow u=b_{0}+a_{1} x+a_{2} y+a_{3} z,
$$

where $a_{1}, a_{2}, a_{3}$ and $b_{0}$ are real constants $\left(a_{1} \neq 0\right)$. Moreover, due to (22), $f=f_{0}=$ const and $g=g_{0}=$ const, and from (16) we have

$$
a_{1}=f_{0} a_{0}, \quad a_{2}=g_{0} a_{0} .
$$

Substituting the above formula for $u$, we reduce the system (4) into

$$
\begin{aligned}
& v_{x}=-f_{0} a_{0}, \quad v_{y}=a_{0}, \quad v_{z}=-f_{0} g_{0} a_{0} \\
& w_{x}=-g_{0} a_{0}, \quad w_{y}=-f_{0} g_{0} a_{0}, \quad w_{z}=a_{0} .
\end{aligned}
$$

Hence

$$
\begin{aligned}
& v=b_{1}-f_{0} a_{0} x+a_{0} y-f_{0} g_{0} a_{0} z, \\
& w=b_{2}-g_{0} a_{0} x-f_{0} g_{0} a_{0} y+a_{0} z,
\end{aligned}
$$

where $b_{1}$ and $b_{2}$ are real constants. Special case $f_{0}=g_{0}=0$ (linear affine functions) was shown to be differentiable in [7], Lemma 4.1.

\section{Exceptional Solutions}

The last case corresponds to $u_{x}=0$. Then Equation (4) yields, immediately, $v_{y}=w_{z}=0$, and, the last equation reads $u_{y} u_{z}=0$. Thus we have two distinct subcases: $u_{y}=0$ (i.e., $u=u(z)$ ) and $u_{z}=0$ (i.e., $u=u(y)$ ).

1. $u=u(y), v=v(x, z), w=w(x, y)$. 
Then $w_{x}=0$ and $u_{y}=-v_{x}$. Hence $u_{y}=c_{0}=$ const and we get the following solution:

$$
u=c_{0} y+c_{1}, \quad v=-c_{0} x+V_{1}(z), \quad w=W_{1}(y),
$$

where $c_{0}$ and $c_{1}$ are constant and $V_{1}=V_{1}(z)$ and $W_{1}=W_{1}(y)$ are arbitrary functions of one variable.

2. $u=u(z), v=v(x, z), w=w(x, y)$.

Then, $w_{x}=-u_{z}$ and $v_{x}=0$. Hence $u_{z}=d_{0}$ and, as a result, we get the solution:

$$
u=d_{0} z+d_{1}, \quad v=V_{2}(y), \quad w=-d_{0} x+W_{2}(z),
$$

where $d_{0}$ and $d_{1}$ are onstant and $V_{2}=V_{2}(y)$ and $W_{2}=W_{2}(z)$ are arbitrary functions of one variable.

Finally, we derived all solutions to the system (4) and thus Theorem 1 is proved.

Author Contributions: Conceptualization, J.L.C.; methodology, J.L.C.; validation, J.L.C. and D.Z.; formal analysis, J.L.C. and D.Z.; investigation, J.L.C. and D.Z.; writing-original draft preparation, J.L.C. and D.Z.; writing-review and editing, J.L.C. All authors have read and agreed to the published version of the manuscript.

Funding: This research received no external funding.

Conflicts of Interest: The authors declare no conflict of interest.

\section{References}

1. Fernández-Guasti, M.; Zaldívar, F. An elliptic non distributive algebra. Adv. Appl. Clifford Algebr. 2013, 23, 825-835. [CrossRef]

2. Kobus, A.; Cieślinnski, J.L. On the Geometry of the Hyperbolic Scator Space in 1+2 Dimensions. Adv. Appl. Clifford Algebr. 2017, 27, 1369-1386. [CrossRef]

3. Cieśliński, J.L.; Kobus, A. On the Product Rule for the Hyperbolic Scator Algebra. Axioms 2020, 9, 55. [CrossRef]

4. Fernández-Guasti, M. Time and space transformations in a scator deformed Lorentz metric. Eur. Phys. J. Plus 2014, 129, 195. [CrossRef]

5. Fernández-Guasti, M. Composition of velocities and momentum transformations in a scator-deformed Lorentz metric. Eur. Phys. J. Plus 2020, 135, 542. [CrossRef]

6. Fernández-Guasti, M. A Non-distributive Extension of Complex Numbers to Higher Dimensions. Adv. Appl. Clifford Algebr. 2015, 25, 829-849. [CrossRef]

7. Fernández-Guasti, M. Differential quotients in elliptic scator algebra. Math. Meth. Appl. Sci. 2018, 41, 4827-4840. [CrossRef]

8. Fernández-Guasti, M. Components exponential scator holomorphic function. Math. Meth. Appl. Sci. 2020, 43, 1017-1034. [CrossRef]

9. Sudbery, A. Quaternionic analysis. Math. Proc. Camb. Philos. Soc. 1979, 85, 199-225. [CrossRef]

10. De Leo, S.; Rotelli, P.P. Quaternionic Analyticity. Appl. Math. Lett. 2003, 16, 1077-1081. [CrossRef]

11. Ryan, J. Clifford analysis. In Lectures on Clifford (Geometric) Algebras and Applications; Abłamowicz, R., Sobczyk, G., Eds.; Birkhäuser, Boston-Basel-Berlin: Basel, Switzerland, 2004; pp. 53-89.

(c) 2020 by the authors. Licensee MDPI, Basel, Switzerland. This article is an open access article distributed under the terms and conditions of the Creative Commons Attribution (CC BY) license (http://creativecommons.org/licenses/by/4.0/). 